九州大学学術情報リポジトリ

Kyushu University Institutional Repository

\title{
Structure-Activity Relationships of Herbicidal Nitrodiphenyl Ethers
}

Ohnishi, Jun-ichi

Laboratory of Pesticide Chemistry, Faculty of Agriculture, Kyushu University

Yukitake, Ken-ichi

Laboratory of Pesticide Chemistry, Faculty of Agriculture, Kyushu University

Eto, Morifusa

Laboratory of Pesticide Chemistry, Faculty of Agriculture, Kyushu University

https://doi.org/10.5109/24015

出版情報：九州大学大学院農学研究院紀要. 37 (3/4)，pp. 239-246，1993-03. Kyushu University バージョン：

権利関係 : 


\title{
Structure-Activity Relationships of Herbicidal Nitrodiphenyl Ethers
}

\author{
Jun-ichi Ohnishi*, Ken-ichi Yukitake**, \\ and Morifusa Eto \\ Laboratory of Pesticide Chemistry, Faculty of Agriculture, \\ Kyushu University 46-02, Fukuoka 812, Japan
}

(Received September 21, 1992)

\begin{abstract}
Thirty 4-(substituted phenoxy) nitrobenzenes were synthesized and examined for phytotoxicity against barnyard-grass in the light and dark. From the structure-activity relationships and light-requisition for the activity, they were classified into three groups. The diphenyl ethers of the first group, which required light for the activity, were characterized by ortho and para substitutions. The second group compounds having meta or ortho and meta substitutions were active even in the dark. The third group involved compounds substituted at both meta and para positions and their herbicidal activity was inhibited by light. p-Amino analog of nitrofen showed high herbicidal activity.
\end{abstract}

\section{INTRODUCTION}

Many $p$-nitrodiphenyl ethers (nitro DPE; I) have been developed to commercial herbicides. Matsunaka (1976) classified them into two groups by their requisition of light for the herbicidal activity. One of the groups requires light for the activity, wheras the other is active even in the dark. The former group including nitrofen (2, 4-dichlorophenyl $p$-nitrophenyl ether; 13) and chlornitrofen (2, 4, 6-trichlorophenyl $p$ nitrophenyl ether; 16) is characterized by having at least one o-substituent on the " $\mathrm{A}$ " benzene ring connecting to the $p$-nitrophenoxy group. The latter group herbicides exemplified by TOPE (p-nitrophenyl m-tolyl ether; 8) have no o-substituent. Major biochemical reactions related to light in plant may be the Hill reaction, photophosphorylation, and photoreduction. Moreland et al. (1970) have examined the effects of light -requiring DPE herbicides involving nitrofen, chlornitrofen, and fluorodifen (2-nitro-4 -trifluoromethylphenyl p-nitrophenyl ether) on these reactions. However they did not test any DPE herbicides of another group. This paper deals with the structureactivity relationships of total 30 nitro DPEs having various pattern of chloro and/or methyl substituents on the " $\mathrm{A}$ " ring. There is evidently an additional group which is inhibited the herbicidal activity by light. The inhibition of the Hill reaction may not be the essential mode of action of light-requiring DPE herbicides.

\footnotetext{
* Present address: Takarazuka Research Center, Sumitomo Chemical Co., Ltd., Takarazuka 665, Japan.

** Present address: Ihara Chemical Co., Ltd., Ikenohata 1-4-26, Taito-ku, Tokyo 110, Japan.
} 


\section{MATERIALS AND METHODS}

\section{Synthesis}

The following two methods were employed for the Williamson synthesis of $p$-nitro DPEs (I).

Method a: To a fused mixture of a substituted phenol (1. 2 mole), KOH (1. 1 mole), and $\mathrm{Al}_{2} \mathrm{O}_{3}(0.05$ mole $)$ at $80-100^{\circ} \mathrm{C}$ was added p-chloronitrobenzene $(1$ mole $)$. The reaction mixture was heated at $145^{\circ} \mathrm{C}$ for $4 \mathrm{hr}$ and then poured into aq. alkaline solution. The precipitate was collected by filtration and recrystallized from ethyl acetate or ethanol. If necessary the product was purified with column chromatography on silicic acid eluted with benzene-hexane mixture (1:3).

Method b: To a mixture. of a substituted phenol (1. 2 mole) and $\mathrm{NaOH}$ (1. 1 mole) in $N, N$-dimethylacetamide at $80-100^{\circ} \mathrm{C}$ was added p-chloronitrobenzene (1 mole). The reaction mixture was heated at $150-160^{\circ} \mathrm{C}$ until p-chloronitrobenzene disappeared and then poured into an aq. alkaline solution. The product was extracted with ether or benzene and purified by recrystallization or silicic acid column chromatography.

2, 4, 6-trichlorophenyl $p$-nitrophenyl ether (chlornitrofen; 16) was a gift from Mitsui-Toatsu Co.

\section{$p$-Aminophenyl 2,4-dichlorophenyl ether}

Nitrofen $(1 \mathrm{~g})$ in benzene $(40 \mathrm{ml})$ was reduced by refluxing in the presence of active iron prepared from iron powder $(10 \mathrm{~g})$ and conc. $\mathrm{HCl}(2 \mathrm{ml})$. Basic fraction extracted with ether was converted to hydrochloride salt, $\mathrm{mp} 133-136^{\circ} \mathrm{C}$. IR: $2800,1580,1500$ $(\mathrm{NH},+)$.

\section{3-(3, 4-Dichlorophenyl)-1, I-dimethylurea (DCMU)}

To 3, 4-dichlorophenyl isocyanate $(10.5 \mathrm{~g})$ in dioxane $(27 \mathrm{ml})$ was dropwise added dimethylamine $(9.13 \mathrm{ml})$ at $20-30^{\circ} \mathrm{C}$. The precipitate was collected by filtration and the filtrate was poured into water to get precipitate as the second crop. The combined products were recrystallized from ethyl acetate, mp $153-4^{\circ} \mathrm{C}$, yield $87 \%$. Anal. Found: C, 46.35; H, 4.29; N, 12.02. Calcd. for $\mathrm{C}_{9} \mathrm{H}_{10} \mathrm{~N}_{2} \mathrm{OCl}_{2}$ : C, 46.20; H, 4.30; N, $11.76 \%$.

\section{Herbicidal Activity}

A test chemical dissolved in acetone was poured onto a sheet of filter paper placed on the bottom of a Petri dish. After evaporation of the solvent, water $(10 \mathrm{ml})$ was poured and 40 seeds of barnyard-grass (Echinochlor crus-galli) were placed in the dish and kept at $30^{\circ} \mathrm{C}$ in the dark or light for 8 days. The light source was two $15 \mathrm{~W}$ fluorescence lamps and the intensity was 700 lux on the surface of the dishes. The length of shoots and roots was measured and compared with that of the control.

\section{Inhibition of Hill Reaction}

Aqueous mixtures $(5.0 \mathrm{ml})$ for the Hill reaction contained spinach chloroplasts containing approximately $80 \mu \mathrm{g}$ of chlorophyll and following chemicals in $\mu$ moles: $\mathrm{pH}$ 8.0 tris buffer, $80 ; \mathrm{NaCl}, 50$; and potassium ferricyanide, 2. Centrifuge tubes containing the reaction mixture were kept in a thermostatted water bath. Reactions were performed at $25^{\circ} \mathrm{C}$ under 20,000 lux irradiated by a $150 \mathrm{~W}$ incandescent reflectorized 
lamp for $15 \mathrm{~min}$ and terminated by the addition of $10 \%$ trichloroacetic acid $(1 \mathrm{ml})$. The precipitate was removed by centrifugation, and ferricyanide reduction in the supernatant was measured at $420 \mathrm{~nm}$. Test chemicals were dissolved in acetone and the final concentration of acetone in all assays including controls was $\mathbf{1 \%}$ by volume. Acetone in this concentration had no detectable effect on the Hill reaction.

\section{RESULTS AND DISCUSSION}

Seven types of p-nitro DPEs were synthesized from substituted phenols and $p^{-}$ chloronitrophenol: 1) o-type (indicating to have substitution(s) only at the ortho positions(s) of "A" ring. Other types are named in similar manner.); 2) m-type; 3) $p$ type; 4) o, p-type; 5) $m$, p-type; 6) o, $m$-type; 7) o, $m$,p -type. The Williamson synthesis for ethers was slightly modifyed by mixing aluminum oxide (method a) or $\mathrm{N}$, N-dimethylacetamide (method b). The structures, physical and analytical data, and method for synthesis of thirty nitro DPEs, except chlornitrofen (16), studied in this paper are listed in Table 1. All the synthesized compounds show appropriate analytical data. Method $\mathrm{b}$ was superior to method a for readiness in separation and purification of products and higher yield. 2, 4, 6-Trimethylphenyl p-nitrophenyl ether (18) could not be synthesized with method a, but b. Methyl-substituted DPEs were synthesized with method $\mathrm{b}$ in relatively short reaction time but the synthesis of chloro derivatives took longer time.

\section{Phyto toxic Activity}

The effects of p-nitro DPEs on the growth of barnyard-grass shoots and roots are summarized in Tables 2 and 3, respectively. Table 2 includes also the half inhibitory molar concentrations $\left(\mathrm{I}_{50}\right)$ for shoot elongation. $p$-NitroDPEs having no substitutent and mono-chloro or methyl group at the ortho or para position of the "A" ring $(1,2$, $3, \mathbf{1 1}, 12)$ showed little activity on the plant growth. o-Type DPEs with plural substitutions (4-6) had only poor activity. o, p-Type derivatives such as 2, 4-dichloro DPE (nitrofen; 13) and 2, 4, 6-trichloro DPE (chlornitrofen; 16) exerted high herbicidal activity in the light but not in the dark. Replacement of a chlorine atom by a methyl group caused decrease in the activity (compare 13 and 16 with 14 and 17, respectively) and compounds having only methyl groups as substituents $(15, \mathbf{1 8})$ were almost inactive. Thus, this type requirs light and chlorine atoms in ortho and para positions for the exertion of herbicidal activity.

All m-type DPEs (7-10) including methyl-substituted compounds exerted high phytotoxicity both under the light and dark conditions. The activity was slightly lower in the light than in the dark. o,m-Type DPEs (24-28) showed similar tendency with m-type DPEs. In this series, however, methyl substituents decrease the activity, except 2,3-dimethyl derivative (25).

In contrast to the above-mentioned types, $m, p$-type DPEs (19-23) were not or weakly active in the light, but considerably active in the dark. Thus, light appears inhibit the phytotoxicity of this type DPEs. Further introduction of another chlorine substituent to the m-positionof 2,4-dichloro DPE (o,p-type 13), the o-position of 3,4dichloro DPE (m,p-type 19), or the p-position of 2,5-dichloro DPE (o, $m$-type 26) made the DPE inactive (29). Another $o, m, p$-type DPE, 30, was also inactive. 
Table 1. Structures and Physical and Analytical Data of $p$-Nitrodiphenyl Ethers (I)

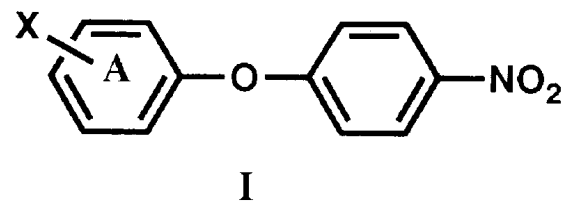

\begin{tabular}{|c|c|c|c|c|c|c|c|c|c|}
\hline \multirow{3}{*}{ No. } & \multirow{3}{*}{$\begin{array}{l}\text { Substituent } \\
\text { X }\end{array}$} & \multirow{3}{*}{$\begin{array}{l}\mathrm{mp} \\
(\text { "C) }\end{array}$} & \multicolumn{6}{|c|}{ Elemental Analysis } & \multirow{3}{*}{ Method } \\
\hline & & & \multicolumn{3}{|c|}{ Found \% } & \multicolumn{3}{|c|}{ Calcd. \% } & \\
\hline & & & $\mathrm{C}$ & $\mathrm{H}$ & $\mathrm{N}$ & $\mathrm{C}$ & $\mathrm{H}$ & $\mathrm{N}$ & \\
\hline 1 & $\mathrm{H}$ & $55-7$ & 66.92 & 4.19 & 6.57 & 66.97 & 4.22 & 6.51 & $\mathrm{a}$ \\
\hline \multicolumn{10}{|c|}{0 -type } \\
\hline 2 & $2-\mathrm{Cl}$ & $75-7$ & 57.68 & 3.29 & 5.62 & 57.73 & 3.23 & 5.61 & $\mathrm{a}$ \\
\hline 3 & ${ }^{2-} \mathrm{Me}$ & $160^{*}$ & 68.13 & 4.86 & 5.97 & 68.11 & 4.84 & 6.11 & $\mathrm{a}$ \\
\hline 4 & $2,6-\mathrm{Cl}_{2}$ & 122 & 50.96 & 2.70 & 4.87 & 50.73 & 2.49 & 4.93 & a \\
\hline 5 & $2-\mathrm{Cl}-6-\mathrm{Me}$ & $80-1$ & 59.19 & 3.80 & 5.23 & 59.21 & 3.83 & 5.35 & $\mathbf{a}$ \\
\hline 6 & $2,6-\mathrm{Me}_{2}$ & $59-60$ & 69.23 & 5.49 & 5.78 & 69.12 & 5.39 & 5.76 & $\mathrm{~b}$ \\
\hline \multicolumn{10}{|c|}{ m-type } \\
\hline 7 & $3-\mathrm{Cl}$ & $58-9$ & 57.62 & 3.10 & 5.51 & 57.73 & 3.23 & 5.61 & $\mathrm{a}$ \\
\hline 8 & 3-Me & $61-2$ & 68.28 & 4.83 & 6.00 & 68.11 & 4.84 & 6.11 & $\mathrm{a}$ \\
\hline 9 & $3,5-\mathrm{Cl}_{2}$ & 80 & 50.68 & 2.48 & 4.90 & 50.73 & 2.49 & 4.93 & $\mathrm{a}$ \\
\hline 10 & $3,5-\mathrm{Me}_{2}$ & $80-1$ & 69.17 & 5.35 & 5.72 & 69.12 & 5.39 & 5.76 & $\mathrm{a}$ \\
\hline \multicolumn{10}{|c|}{$p$-type } \\
\hline 11 & $4-\mathrm{Cl}$ & $75-6$ & 57.71 & 3.15 & 5.41 & 57.73 & 3.23 & 5.61 & a \\
\hline 12 & $4-\mathrm{Me}$ & $66-7$ & 68.16 & 4.84 & 6.24 & 68.11 & 4.84 & 6.11 & a \\
\hline \multicolumn{10}{|c|}{$o, p$-type } \\
\hline 13 & $2,4-\mathrm{Cl}_{2}$ & 71 & 50.67 & 2.48 & 4.90 & 50.73 & 2.49 & 4.93 & $\mathrm{a}$ \\
\hline 14 & $2-\mathrm{Me}^{-4}-\mathrm{Cl}$ & $61-3$ & 59.40 & 3.81 & 5.36 & 59.21 & 3.83 & 5.35 & $\mathrm{a}$ \\
\hline 15 & $2,4-\mathrm{Me}_{2}$ & $59-61$ & 69.20 & 5.37 & 5.77 & 69.12 & 5.39 & 5.76 & $\mathrm{a}$ \\
\hline 16 & $2,4,6-\mathrm{Cl}_{3}$ & $105-6$ & & & - & - & - & - & \\
\hline 17 & $2,4-\mathrm{Cl}_{2}-6-\mathrm{Me}$ & $93-4$ & 52.53 & 3.08 & 4.63 & 52.37 & 3.05 & 4.70 & a \\
\hline 18 & $2,4,6-\mathrm{Me}_{3}$ & $86-7$ & 70.16 & 5.98 & 5.50 & 70.02 & 5.88 & 5.44 & $\mathrm{~b}$ \\
\hline \multicolumn{10}{|c|}{$m, p$-type } \\
\hline 19 & $3,4-\mathrm{Cl}_{2}$ & $79-80$ & 50.84 & 2.68 & 4.68 & 50.73 & 2.49 & 4.93 & $\mathrm{a}$ \\
\hline 20 & $3-\mathrm{Me}^{-4}-\mathrm{Cl}$ & 111 & 59.36 & 3.86 & 5.26 & 59.21 & 3.83 & 5.35 & $\mathrm{a}$ \\
\hline 21 & $3,4-\mathrm{Me}_{2}$ & $87-8$ & 69.19 & 5.37 & 5.73 & 69.12 & 5.39 & 5.76 & a \\
\hline 22 & $3,4,5-\mathrm{Me}_{3}$ & $100-1$ & 70.01 & 5.69 & 5.46 & 70.02 & 5.88 & 5.44 & a \\
\hline 23 & $3,5-\mathrm{Me}_{2}-4-\mathrm{Cl}$ & 121 & 60.57 & 4.36 & 5.00 & 60.54 & 4.36 & 5.04 & a \\
\hline \multicolumn{10}{|c|}{ o,m-type } \\
\hline 24 & $2,3-\mathrm{Cl}_{2}$ & 123 & 50.74 & 2.48 & 4.96 & 50.73 & 2.49 & 4.93 & b \\
\hline 25 & $2,3-\mathrm{Me}_{2}$ & $76-7$ & 69.16 & 5.40 & 5.78 & 69.12 & 5.39 & 5.76 & b \\
\hline 26 & $2,5-\mathrm{Cl}_{2}$ & $95-6$ & 50.74 & 2.49 & 4.87 & 50.73 & 2.49 & 4.93 & b \\
\hline 27 & $2,5-\mathrm{Me}_{2}$ & $128^{* *}$ & 68.95 & 5.44 & 5.76 & 69.12 & 5.39 & 5.76 & $\mathrm{a}$ \\
\hline 28 & $2,3,5-\mathrm{Me}_{3}$ & $98-9$ & 70.29 & 6.02 & 5.61 & 70.02 & 5.88 & 5.44 & b \\
\hline \multicolumn{10}{|c|}{$o, m, p$-type } \\
\hline 29 & $2,4,5-\mathrm{Cl}_{3}$ & 115 & 45.47 & 2.17 & 4.41 & 45.42 & 1.91 & 4.41 & $\mathrm{~b}$ \\
\hline 30 & $2,4,5-\mathrm{Me}_{3}$ & $72-3$ & 70.07 & 5.84 & 5.44 & 70.02 & 5.88 & 5.44 & $\mathrm{~b}$ \\
\hline
\end{tabular}

*bp at $3 \mathrm{mmHg} \quad * *$ bp at $0.2 \mathrm{mmHg}$ 
Table 2. Effect of p-Nitrodiphenyl Ethers (I) on Growth of Barnyard-grass Shoots.

\begin{tabular}{|c|c|c|c|c|c|c|c|c|c|c|c|}
\hline \multirow[b]{3}{*}{ No. } & \multirow[b]{3}{*}{$\mathrm{X}$} & \multicolumn{8}{|c|}{ Average length of shoots ( $\%$ of control) } & & \\
\hline & & \multicolumn{2}{|c|}{ 10ppm } & \multicolumn{2}{|c|}{$50 \mathrm{ppm}$} & \multicolumn{2}{|c|}{$100 \mathrm{ppm}$} & \multicolumn{2}{|c|}{$200 \mathrm{ppm}$} & \multicolumn{2}{|c|}{$\mathrm{I}_{50}\left(\mathrm{x} 10^{-5} \mathrm{M}\right)$} \\
\hline & & $\mathrm{L}$ & $\mathrm{D}$ & $\mathrm{L}$ & $\mathrm{D}$ & $\mathrm{L}$ & $\mathrm{D}$ & $\mathrm{L}$ & $\mathrm{D}$ & $\mathrm{L}$ & $\mathrm{D}$ \\
\hline 1 & $\mathbf{H}$ & 112 & 67 & 111 & 10 & & 14 & 84 & -- & $-i$ & - \\
\hline \multicolumn{12}{|c|}{ o-type } \\
\hline 2 & $2-\mathrm{Cl}$ & 108 & 108 & 91 & 90 & 81 & 73 & - & - & & \\
\hline 3 & $2-\mathrm{Me}$ & 123 & 79 & 91 & 100 & 114 & 90 & - & - & & \\
\hline 4 & $2,6-\mathrm{Cl}_{2}$ & & - & 67 & 109 & 78 & 74 & 52 & 116 & & \\
\hline 5 & $2-\mathrm{Cl}-6-\mathrm{Me}$ & & - & 78 & 95 & 70 & 74 & 65 & 63 & & \\
\hline 6 & $2,6-\mathrm{Me}_{2}$ & & - & 81 & 84 & 89 & 84 & 74 & 65 & - & - \\
\hline \multicolumn{12}{|c|}{ m-type } \\
\hline 7 & 3-Cl & 62 & 50 & 15 & 0 & 0 & 0 & - & - & 7.2 & 4.0 \\
\hline 8 & 3-Me & 66 & 55 & 0 & 0 & 0 & 0 & - & - & 6.1 & 4.8 \\
\hline 9 & $3,5-\mathrm{Cl}^{*}$ & 87 & 52 & 40 & 0 & 0 & 12 & - & - & 14.0 & 3.7 \\
\hline 10 & $3,5-\mathrm{Me}_{2}$ & 59 & 5 & 18 & 0 & 33 & 0 & - & - & 6.4 & 1.2 \\
\hline \multicolumn{12}{|c|}{$p$-typ } \\
\hline 11 & 4-Cl & 101 & 86 & 67 & 100 & 77 & 74 & - & - & & \\
\hline 12 & 4-Me & 110 & 100 & 98 & 72 & 74 & 73 & - & - & - & - \\
\hline \multicolumn{12}{|c|}{$o, p$-type } \\
\hline 13 & $2,4-\mathrm{Cl}_{2}$ & 62 & 100 & 27 & 96 & 22 & 93 & - & - & 7.0 & - \\
\hline 14 & $2-\mathrm{Me}^{-4}-\mathrm{Cl}$ & 116 & 84 & 76 & 98 & 63 & 88 & - & - & 102.0 & - \\
\hline 15 & $2,4-\mathrm{Me}_{2}$ & 94 & 102 & 97 & 92 & 86 & 89 & - & - & - & - \\
\hline 16 & $2,4,6-\mathrm{Cl}_{3}$ & 64 & 110 & 23 & 89 & 15 & 92 & - & - & 6.0 & - \\
\hline 17 & $2,4-\mathrm{Cl}_{2}-6-\mathrm{Me}$ & 79 & 98 & 70 & 102 & 60 & 105 & - & - & 50.0 & - \\
\hline 18 & $2,4,6-\mathrm{Me}_{3}$ & & - & 81 & 88 & 97 & 126 & 88 & 92 & & \\
\hline \multicolumn{12}{|c|}{$m, p$-type } \\
\hline 19 & $3,4-\mathrm{Cl}_{2}$ & 82 & 52 & 62 & 28 & 55 & 11 & - & - & 57.0 & 4.6 \\
\hline 20 & 3-Me-4-U & 75 & 82 & 73 & 37 & 69 & 0 & - & - & - & 14.0 \\
\hline 21 & $3,4-\mathrm{Me}_{2}$ & 92 & 65 & 86 & 18 & 81 & 16 & - & - & - & 5.8 \\
\hline 22 & $3,4,5-\mathrm{Me}_{3}$ & 88 & 47 & 102 & 12 & 89 & 0 & - & - & - & 3.1 \\
\hline 23 & $3,5-\mathrm{Me}_{2}-4-\mathrm{Cl}$ & 104 & 62 & 80 & 4 & 105 & 0 & - & - & - & 4.3 \\
\hline \multicolumn{12}{|c|}{$0, m$-type } \\
\hline 24 & $2,3-\mathrm{Cl}_{2}$ & 79 & 79 & 52 & 0 & 48 & 0 & - & - & 21.5 & 9.9 \\
\hline 25 & $2,3-\mathrm{Me}_{2}$ & 98 & 54 & 51 & 0 & 21 & 0 & - & - & 18.1 & 4.5 \\
\hline 26 & $2,5-\mathrm{Cl}_{2}$ & 86 & 63 & 64 & 44 & 52 & 37 & 51 & 28 & 44.0 & 12.3 \\
\hline 27 & 2,5-Me, & - & - & 91 & 76 & 93 & 79 & 85 & 64 & & \\
\hline 28 & $2,3,5-\mathrm{Me}_{3}$ & & & & - & 101 & 110 & 99 & 97 & - & - \\
\hline \multicolumn{12}{|c|}{$o, m, p-$ type } \\
\hline 29 & $2,4,5-\mathrm{Cl}_{3}$ & & & & - & 104 & 82 & 80 & 72 & & \\
\hline 30 & $2,4,5-\mathrm{Me}_{3}$ & - & - & - & - & 110 & 126 & 104 & 90 & & \\
\hline
\end{tabular}

L: light D: dark

The present relationships of $o, p$ - and m-type DPEs to light in phytotoxicity exertation support Matsunaka's classification of DPEs in which the light-requiring DPEs have at least one o-substituent on the "A" benzene ring (Matsunaka, 1976). However, o,m-type DPEs are out of this classification. They have an o-substituent, but require no light for the activity. Thus, the light-requiring DPEs should have substituents at both $o-$ and p-positions. $m, p$-Type DPEs whose phytotoxicity was inhibited by light should be classified into additional new group. 
p-Aminophenyl 2,4-dichlorophenyl ether, which may be formed from nitrofen by photochemical or metabolic reduction in plants, almost completely killed barnyardgrass at $500 \mathrm{ppm}$ and inhibit the growth more than $80 \%$ at $100 \mathrm{ppm}$ under the dark conditions. Nitrofen itself did not show any effect to the growth of barnyard-grass at $500 \mathrm{ppm}$ under the dark conditions.

\section{Inhibition of Hill Reaction}

The inhibitory activity of $p$-nitro DPEs toward the Hill reaction is listed in Table

Table 3. Effect of $p$-Nitrodiphenyl Ethers (I) on Growth of Barnyard-grass Roots.

\begin{tabular}{|c|c|c|c|c|c|c|c|c|c|}
\hline \multirow[b]{3}{*}{ No. } & \multirow[b]{3}{*}{$\mathrm{X}$} & \multicolumn{8}{|c|}{ Average length of roots ( $\%$ of control) } \\
\hline & & \multicolumn{2}{|c|}{$10 \mathrm{ppm}$} & \multicolumn{2}{|c|}{$50 \mathrm{ppm}$} & \multicolumn{2}{|c|}{$100 \mathrm{ppm}$} & \multicolumn{2}{|c|}{$200 \mathrm{ppm}$} \\
\hline & & $\mathrm{L}$ & $\mathrm{D}$ & $\mathrm{L}$ & $\mathrm{D}$ & $\mathrm{L}$ & $\mathrm{D}$ & $\mathrm{L}$ & $\mathrm{D}$ \\
\hline 1 & & 85 & 135 & 83 & 129 & 84 & 89 & - & - \\
\hline \multicolumn{10}{|c|}{0 -type } \\
\hline 2 & $2-\mathrm{Cl}$ & 118 & 114 & 114 & 87 & 76 & 65 & - & - \\
\hline 3 & $2-\mathrm{Me}$ & 105 & 62 & 105 & 93 & 110 & 133 & - & \\
\hline 4 & $2,6-\mathrm{Cl}_{2}$ & & - & 111 & 106 & 110 & 62 & 105 & 86 \\
\hline 5 & $2-\mathrm{Cl}-6-\mathrm{Me}$ & & - & 117 & 101 & 105 & 120 & 99 & 88 \\
\hline 6 & $2,6-\mathrm{Me}_{2}$ & - & - & 97 & 101 & 87 & 110 & 97 & 106 \\
\hline \multicolumn{10}{|c|}{ m-type } \\
\hline 7 & $3-\mathrm{Cl}$ & 35 & 32 & 11 & 0 & 0 & 0 & - & - \\
\hline 8 & $3-\mathrm{Me}$ & 66 & 106 & 0 & 0 & 0 & 0 & - & - \\
\hline 9 & $3,5-\mathrm{Cl}_{2}$ & 84 & 46 & 8 & 0 & 0 & 0 & - & - \\
\hline 10 & $3,5-\mathrm{Me}_{2}$ & 71 & 10 & 6 & 0 & 5 & 0 & - & - \\
\hline \multicolumn{10}{|c|}{$p$-type } \\
\hline 11 & $4-\mathrm{Cl}$ & 112 & 118 & 42 & 99 & 69 & 110 & - & - \\
\hline 12 & $4-\mathrm{Me}$ & 136 & 127 & 121 & 122 & 109 & 94 & - & \\
\hline \multicolumn{10}{|c|}{$o, p$-type } \\
\hline 13 & $2,4-\mathrm{Cl}_{2}$ & 94 & 98 & 26 & 100 & 24 & 117 & - & - \\
\hline 14 & $2-\mathrm{Me}^{-4}-\mathrm{Cl}$ & 134 & 53 & 77 & 98 & 90 & 101 & - & \\
\hline 15 & $2,4-\mathrm{Me}_{2}$ & 121 & 135 & 120 & 51 & 114 & 108 & - & - \\
\hline 16 & $2,4,6-\mathrm{Cl}_{3}$ & 67 & 100 & 11 & 71 & 9 & 93 & - & - \\
\hline 17 & $2,4-\mathrm{Cl}_{2}-6-\mathrm{Me}$ & 123 & 126 & 63 & 82 & 71 & 69 & - & \\
\hline 18 & $2,4,6-\mathrm{Me}_{3}$ & & - & 109 & 26 & 108 & 77 & - & - \\
\hline \multicolumn{10}{|c|}{$m, p$-type } \\
\hline 19 & $3,4-\mathrm{Cl}_{2}$ & 50 & 47 & 12 & 20 & 89 & 0 & - & - \\
\hline 20 & $3-\mathrm{Me}^{-4}-\mathrm{Cl}$ & 113 & 91 & 23 & 34 & 47 & 0 & - & - \\
\hline 21 & $3,4-\mathrm{Me}_{2}$ & 79 & 56 & 50 & 25 & 48 & 12 & - & \\
\hline 22 & $3,4,5-\mathrm{Me}_{3}$ & 93 & 50 & 61 & 11 & 71 & 0 & - & \\
\hline 23 & $3,5-\mathrm{Me}_{2}-4-\mathrm{Cl}$ & 108 & 82 & 62 & 40 & 61 & 0 & - & - \\
\hline \multicolumn{10}{|c|}{ o,m-type } \\
\hline 24 & $2,3-\mathrm{Cl}_{2}$ & 52 & 40 & 31 & 0 & 27 & 0 & - & - \\
\hline 25 & $2,3-\mathrm{Me}_{2}$ & 110 & 62 & 35 & 0 & 15 & 0 & - & \\
\hline 26 & $2,5-\mathrm{Cl}_{2}$ & 73 & 23 & 32 & 8 & 29 & 22 & 43 & 15 \\
\hline 27 & $2,5-\mathrm{Me}_{2}$ & - & - & 135 & 101 & 111 & 76 & 161 & 49 \\
\hline 28 & $2,3,5-\mathrm{Me}_{3}$ & $\overline{-}$ & - & - & - & 121 & 128 & 175 & 122 \\
\hline \multicolumn{10}{|c|}{$o, m, p$-type } \\
\hline 29 & $2,4,5-\mathrm{Cl}_{3}$ & - & - & - & - & 109 & 70 & 43 & 75 \\
\hline 30 & $2,4,5-\mathrm{Me}_{3}$ & - & - & - & - & 100 & 159 & 145 & 107 \\
\hline
\end{tabular}

L: light D: dark 
4. Only the weak activity was found in P-type DPEs which showed no phytotoxicity, $m, p$-type DPEs which were not phytotoxic in the light, and m-type DPEs which were phytotoxic independently of light. In contrary the $o, p$-type DPEs which require light for phytotoxicity showed the high inhibitory activity. However, difference in the inhibitory activity between the $0, p$-type and the three other types is not very distinctive.

On the other hand, o,m-type DPEs which were classified into the same group as m-type, exerting herbicidal activity both in the light and dark, inhibited the Hill reaction more strongly than m-type DPEs. Furthermore, both $o$ - and $o, m$, $p$-type DPEs which were non-phytotoxic rather strongly inhibited the Hill reaction. The

Table 4. Inhibition of Hill Reaction by P-Nitrodiphenyl Ethers (I).

\begin{tabular}{|c|c|c|c|}
\hline No. & $\mathrm{X}$ & $\begin{array}{l}\text { Inhibition \% } \\
\left(\text { at } 10^{-4} \mathrm{M}\right)\end{array}$ & $\left(\begin{array}{c}\mathrm{I}_{50} \\
\left(\times 10^{-5} \mathrm{M}\right)\end{array}\right.$ \\
\hline 1 & $\mathbf{H}$ & 39 & - \\
\hline \multicolumn{4}{|c|}{0 -type } \\
\hline 2 & $2-\mathrm{Cl}$ & 31 & - \\
\hline 3 & $\mathrm{Z}-\mathrm{Me}$ & 42 & \\
\hline 4 & $2,6-\mathrm{Cl}_{2}$ & 56 & 7.7 \\
\hline 5 & $2-\mathrm{Cl}-6-\mathrm{Me}$ & 60 & 8.0 \\
\hline 6 & $2,6-\mathrm{Me}_{2}$ & 59 & 8.3 \\
\hline \multicolumn{4}{|c|}{ m-type } \\
\hline 7 & $3-\mathrm{Cl}$ & 41 & - \\
\hline 8 & $3-\mathrm{Me}$ & 35 & - \\
\hline 9 & $3,5-\mathrm{Cl}_{2}$ & 45 & - \\
\hline 10 & $3,5-\mathrm{Me}_{2}$ & 39 & - \\
\hline \multicolumn{4}{|c|}{$p$-type } \\
\hline 11 & $4-\mathrm{Cl}$ & 47 & - \\
\hline 12 & 4-Me & 45 & - \\
\hline \multicolumn{4}{|c|}{$o, p$-type } \\
\hline 13 & $2,4-\mathrm{Cl}_{2}$ & 66 & 7.7 \\
\hline 14 & $2-\mathrm{Me}^{-4}-\mathrm{Cl}$ & 55 & 8.8 \\
\hline 15 & $2,4-\mathrm{Me}_{2}$ & 61 & 8.3 \\
\hline 16 & $2,4,6-\mathrm{Cl}_{3}$ & 77 & 4.5 \\
\hline 17 & $2,4-\mathrm{Cl}_{2}-6-\mathrm{Me}$ & 72 & 7.0 \\
\hline 18 & $2,4,6-\mathrm{Me}_{3}$ & 73 & 5.6 \\
\hline \multicolumn{4}{|c|}{$m, p$-type } \\
\hline 19 & $3,4-\mathrm{Cl}_{2}$ & 38 & - \\
\hline 20 & $3-\mathrm{Me}^{-4}-\mathrm{Cl}$ & 37 & - \\
\hline 21 & $3,4-\mathrm{Me}_{2}$ & 36 & - \\
\hline 22 & $3,4,5-\mathrm{Me}_{3}$ & 48 & - \\
\hline 23 & $3,5-\mathrm{Me}_{2}-4-\mathrm{Cl}$ & 44 & - \\
\hline \multicolumn{4}{|c|}{0, m-type } \\
\hline 24 & $2,3-\mathrm{Cl}_{2}$ & 56 & 8.5 \\
\hline 25 & $2,3-\mathrm{Me}_{2}$ & 60 & 7.1 \\
\hline 26 & $2,5-\mathrm{Cl}_{2}$ & 66 & 6.7 \\
\hline 27 & $2,5-\mathrm{Me}_{2}$ & 67 & 8.0 \\
\hline 28 & $2,3,5-\mathrm{Me}_{3}$ & 61 & 5.6 \\
\hline \multicolumn{4}{|c|}{$o, m, p$-type } \\
\hline 29 & $2,4,5-\mathrm{Cl}_{3}$ & 68 & 1.0 \\
\hline 30 & $2,4,5-\mathrm{Me}_{3}$ & 64 & 6.0 \\
\hline
\end{tabular}


inhibitory activity of the present DPEs, even the most active one, was several hundreds time less than that of the herbicide DCMU, the well known Hill reaction inhibotor. In view of these data, the inhibition of the Hill reaction is not considered as the essential mode of action of the light-requiring DPE herbicides.

An action mechanism for the light-requiring DPE herbicides has been presented by studies on acifluorfen-methyl [methyl 5-(2-chloro-4-trifluoromethylphenoxy)-2nitrobenzoate] and related DPEs that they accumulate tetrapyrroles such as protoporphyrin IX and protochlorophyllide by stimulating 5-aminolevulinic acid synthesis and tetrapyrroles-sensitized photoperoxidation of membrane lipids causes eventual death of plant cells (Duke et al., 1991; Haworth and Dan Hess, 1988; Kouji et al., 1988; Matringe and Scalla, 1988). The action mechanism for other DPEs which do not require light for the herbicidal activity must be studied in other direction.

\section{REFERENCES}

Duke, S. O., J. M. Becerril, T. D. Sherman and H. Matsumoto 1991 Photosensitizing porphyrins as herbicides. In "Naturally Occurring Pest Bioregulators", ACS Symposium Series 449, ed. by P. A. Hedin, American Chemical Society, Washington, pp 371-386

Haworth, P. and F. Dan Hess 1988 The generation of singlet oxygen $\left({ }^{1} \mathrm{O}_{2}\right)$ by the nitrodiphenyl ether herbicide oxyfluorfen is independent of photosynthesis. Plant Physiol., 86: 672-676

Kouji, H., T. Masuda and S. Matsunaka 1988 Action mechanism of diphenyl ether herbicides: Light-dependent $\mathrm{O}_{2}$ consumption in diphenyl ether-treated tobacco cell homogenate. J. Pesticide Sci., 13: 495-499.

Matringe, M. and R. Scalla 1988 Studies on the mode of action of acifluorfen-methyl in nonchlorophyllous soybean cells. Plant Physiol., 86: 619-622

Matsunaka, S. 1976 Diphenyl ethers. In "Herbicides Chemistry, Degradation, and Mode of Action”, Vol. 2, ed. by P. C. Keamey and D. D. Kaufman, Marcel Dekker, New York, pp. 709-739

Moreland, D. E., W. J. Blackmon. H. G. Todd and F. S. Farmer 1970 Effects of diphenylether herbicides on reactions of mitochondria and chloroplasts. Weed Science, 18: 636-642 